nales de salud para reforzar el compromiso político necesario para avanzar en la eliminación de la tuberculosis en los países de baja prevalencia.

\section{Bibliografía}

1.- GONZÁLEZ E, ARMAS L. Eliminación de la tuberculosis como problema de salud pública: una elección acertada. Rev Esp Salud Pública 2007; 81: 59-62.

2.- OFICINA REGIONAL PARA EL CONTROL DE LA TUBERCULOSIS EN LAS AMÉRICAS. Agenda de la VI Reunión Regional de Países de Baja prevalencia de Tuberculosis en Las Américas. Organización Panamericana de la Salud. Santiago de Chile 2013.
3.- DEL GRANADO M. Objetivos y resultados esperados de la reunión. Santiago de Chile, 2013.

4.- DEL GRANADO M. Control de la tuberculosis en los países de baja prevalencia. Santiago de Chile 2013.

5.- RODRÍGUEZ J. Presentación de Uruguay como país de baja prevalencia. Santiago de Chile 2013.

6.- MATA Z. Presentación de Costa Rica como país de baja prevalencia. Santiago de Chile, 2013.

7.- LLANES M J. Presentación de Cuba como país de baja prevalencia. Santiago de Chile 2013.

8.- HERRERA T. Presentación de Chile como país de baja prevalencia. Santiago de Chile 2013.

9.- DEL GRANADO M. Conclusiones y recomendaciones de la VI Reunión de países de baja prevalencia. Santiago de Chile 2013.

\title{
Comentarios sobre los países de baja prevalencia de tuberculosis en América Latina
}

\author{
ÁLVARO YÁÑEZ DEL V.*
}

\author{
Commentaries on the epidemiological situation of Latin American countries \\ of low prevalence of tuberculosis
}

\section{Situación}

Chile, Costa Rica, Cuba y Uruguay, han logrado una reducción de la endemia tuberculosa a tasas inferiores a 20 casos por 100.000 habitantes, tasa que corresponde al umbral de eliminación de la tuberculosis como problema de Salud Pública, condición epidemiológica que se consideró posible alcanzar y que abría la posibilidad cierta de lograr la eliminación de la enfermedad en el largo plazo. Actualmente se considera que la enfermedad se ha eliminado de una comunidad, cuando la incidencia llega a 1 caso o menos por 1.000.000 habitantes.

Si bien con las tasas actuales la mayor parte de la población en estos países tiene un riesgo muy bajo de ser infectada y enfermar, en todos ellos existen grupos más o menos circunscritos, con un riesgo varias veces superior al del promedio nacional, constituyendo estos sectores vulnerables, junto con la población mayor de 60 años, el último reducto de la enfermedad. A lo anterior se suma en estos países, en los últimos años, la detención del proceso de reducción de la incidencia, lo cual determina un exceso de morbilidad en relación a lo esperado de haberse mantenido la tendencia observada en la última década.

Esta es una poderosa señal de alarma, que pone en evidencia que se mantiene la cadena de transmisión de la enfermedad, no continúa la reducción de la incidencia de la morbilidad y se mantiene la mortalidad, sin descender a su mínima expresión.

\section{Amenaza}

En caso de mantenerse esta situación por un tiempo superior a 4 ó 5 años, se puede comenzar a asistir a la reversión epidemiológica de la endemia de la tuberculosis, con ascenso de la incidencia, aumento de la prevalencia, incremento del número de fracasos, mortalidad por la enfermedad y surgimiento de casos tuberculosis

* Asesor Programa de Control de la Tuberculosis, Ministerio de Salud, Chile y ex Asesor Regional de Tuberculosis de la Organización Panamericana de la Salud. 
multidrogorresistente (TB-MDR) y tuberculosis extremadamente drogorresistente (TB-XDR) ${ }^{1}$. Además, se retrocede a una condición epidemiológica superada hace décadas y se posterga la posibilidad de eliminar la tuberculosis como problema de Salud Pública.

Es posible que en el futuro investigaciones en curso permitirán disponer de nuevos recursos para utilizarlos en conjunto: Una vacuna capaz de impedir la infección o re-infección, así como el paso de la infección a enfermedad; quimioterapia eficaz para eliminar bacilos persistentes y acabar con la tuberculosis latente, y esquemas de tratamiento de mayor eficacia, con mejor tolerancia, ausencia de efectos colaterales graves y menor duración.

De nada de esto se dispone en la actualidad; por lo tanto se deben identificar las causas de la situación señalada, reforzar el Programa de Control de la Tuberculosis (PCT), optimizar el uso de los recursos diagnósticos y de tratamiento disponibles en la actualidad y, cuando sea posible, reforzarlos con la incorporación de nuevos métodos para la localización, diagnóstico y tratamiento de la tuberculosis.

\section{Policausalidad de la situación descrita}

\section{La situación actual, estado de los conocimientos, recursos tecnológicos y operacionales e idoneidad de los responsables directos del PCT, no son la causa de esta situación}

No hay modificaciones recientes y graves de las condiciones generales en estos países, sean de orden económico o derivadas de graves crisis sociales o institucionales, que hayan contribuido a empeorar la condición general de la población $\mathrm{y}$ al deterioro de la atención de salud en general y de la aplicación del PCT en especial.

Los fundamentos conceptuales del PCT vigente en estos países, así como sus normas técnicas $\mathrm{y}$ administrativas son adecuados y conforme a las recomendaciones internacionales. La tecnología disponible, cuando fue aplicada en forma eficiente, fue capaz de determinar una sostenida y satisfactoria reducción de la incidencia de la enfermedad. La idoneidad de los responsables y dirigentes directos del PCT en estos países, es de buen nivel. Por tanto, no es en estos aspectos

\footnotetext{
${ }^{1} \mathrm{~TB}-\mathrm{MDR}$ es aquella con resistencia a isoniacida y rifampicina; TB XDR corresponde a una TB-MDR que además es resistente a flouroquinolonas y un aminoglicósido de segunda línea.
}

donde se pueden encontrar las causas de esta situación, ni el camino para corregirla.

\section{Reducción del recurso financiero}

Pero, al menos en dos de estos países, han ocurrido reducciones drásticas de presupuesto, que han afectado la capacidad para mantener actividades de capacitación y apoyo al PCT, asegurar la supervisión y actividades de evaluación, efectuar el mantenimiento de sus instalaciones, en especial las de laboratorio, donde no ha sido posible la adquisición de equipamiento que permita la incorporación de nuevas tecnologías para mejorar la localización, diagnóstico y control del tratamiento y, en la atención directa de casos, aplicar medidas que hagan posible lograr alta eficiencia y eficacia del tratamiento.

Está absolutamente justificado insistir en la necesidad de disponer de los recursos necesarios para asegurar una gestión y aplicación operacionalmente significativa y eficiente del Programa.

Pero, sería un error limitar las causas exclusivamente a la falta de recursos. La ineficiencia en la localización de casos, con diagnóstico retardado de las fuentes de contagio, no se debe a la falta de recursos. La alta tasa de abandonos y muertes en el curso del tratamiento, tampoco se deben al desabastecimiento de medicamentos o falta de cobertura asistencial.

\section{Falta de Apoyo Administrativo}

Lo que indudablemente ha agravado la situación actual es la falta o débil apoyo administrativo que autoridades de salud de todo nivel ofrecen al PCT.

Reiteradas medidas de intervención, efectuadas a veces con gran esfuerzo y sacrificio personal de los encargados del Programa, como son actividades de supervisión y capacitación y la elaboración y distribución de directrices operacionales, no han producido el efecto deseado, al no contar con el apoyo administrativo que haga posible la aplicación de actividades absolutamente necesarias para el éxito del Programa.

Las causas de esta falta de apoyo administrativo, son de orden sociológico, culturales y políticas, como por ejemplo la presión que existe sobre los directivos para dar respuesta a las demandas de atención con insuficientes recursos, la obligación de productividad asistencial y en ocasiones la pobre calidad de liderazgo a nivel de los equipos de salud del nivel local.

Finalmente, de importancia determinante, han sido las características de la orientación y conducción político-administrativa de la salud en el país, cuya acción negativa ha sido capaz de poner 
en peligro incluso la existencia misma del PCT o por lo menos ha afectado seriamente su eficiencia. Los factores causales de estos condicionantes se deben identificar con claridad. Algunos de ellos se analizan a continuación.

\section{Pérdida de trascendencia de la enfermedad}

Se observa una pérdida de la trascendencia social de la tuberculosis, por la menor frecuencia del diagnóstico y muerte por esta causa. La mayoría de las familias hoy no tienen ningún miembro que haya sufrido tuberculosis en época reciente $\mathrm{y}$, menos aún, algún fallecido por esta causa.

Rara vez la enfermedad es mencionada en los medios de comunicación y nunca se hace referencia a su importancia como problema de Salud Pública. Hay escasa o nula presencia de ella en los medios informativos y su relación con la pobreza y la inequidad social y el sufrimiento humano que provoca, no constituyen noticia. De hecho no hay conciencia social de qué se trata cuando se habla de tuberculosis como un problema de Salud Pública.

Tuberculosis no es un tema importante en las Escuelas de Medicina y de otras carreras de la salud, nunca hay mención de la epidemiología, ni de los aspectos técnicos, operacionales y administrativos que constituyen el Programa de control de la enfermedad.

En ocasiones, el interés docente se centra en la descripción clínica del caso pulmonar avanzado, lo que constituye la evidencia del diagnóstico tardío, ignorando que el problema que se debe resolver es el diagnóstico en la fase inicial de la enfermedad.

En las clases de medicina preventiva de pregrado y en las escuelas de Salud Pública, no siempre se tiene la información epidemiológica actualizada sobre tuberculosis, ni se explican los fundamentos racionales, tanto de las medidas de control de la enfermedad como las de su eliminación como problema de Salud Pública.

\section{Desconocimiento general de la tuberculosis}

Hay desconocimiento en la población e incluso en el personal de salud, sobre la enfermedad y la situación epidemiológica actual. No se le concede toda su importancia a los casos pulmonares confirmados por bacteriología y se ignora la racionalidad de las medidas de control, como la localización por tamizaje con la bacteriología como herramienta de decisión diagnóstica y el control del tratamiento y las razones por las cuales éste debe efectuarse bajo observación directa.

En los niveles de toma de decisión política a nivel Gobierno y de conducción administrativa de los diversos niveles de la estructura de salud, hay falta de información y conocimiento epidemiológico actualizado sobre la enfermedad y sobre la cuantía de los recursos requeridos para la aplicación eficiente de las medidas de control.

\section{Falta de una Política de Estado explícita en relación a la eliminación de la tuberculosis como problema de Salud Pública}

Debido a lo anterior, no se le ha dado categoría de política de estado en salud al propósito de cumplir la meta sanitaria de eliminar la tuberculosis como problema de salud pública (reducir la incidencia a 5 ó menos casos/100.000, para el año 2020) y eliminar la tuberculosis (menos de 1 caso por millón de habitantes, para el año 2050).

$\mathrm{Al}$ no haber una política de estado al respecto, los sucesivos gobiernos han carecido de la voluntad política para otorgar el apoyo administrativo y asignar los recursos necesarios para mantener la eficiencia del PCT, requisito indispensable para cumplir las metas señaladas. Peor aún, en la contingencia, es frecuente restar recursos y personal al PCT, para atender necesidades que se estiman más apremiantes.

\section{Falta de compromiso e interés del Personal de Salud en el PCT}

Aunque parte de los profesionales y personal auxiliar involucrados directamente en las actividades del Programa, cumplen satisfactoriamente sus actividades, el resto, quizás la mayoría, las cumple como rutina, frecuentemente en forma inadecuada y sin esforzarse en mejorar su trabajo. El personal no involucrado directamente, sencillamente no colabora en aplicar las normas del PCT en su ámbito de trabajo, en especial con la pesquisa en los sintomáticos respiratorios.

\section{Percepción individual sobre la enfermedad}

Existe baja percepción individual sobre la presencia de enfermedad, en especial en las fases incipientes de la tuberculosis, situación que puede prolongarse por meses, sin que el enfermo le conceda atención, hasta que el compromiso del estado general y la intensidad de los síntomas la hace evidente. Si no hay interés en el personal de salud en precisar la condición de sintomático respiratorio, la aplicación de la estrategia de localización de casos mediante el tamizaje será deficiente.

Estos componentes contribuyen a provocar demora en el diagnóstico de las fuentes de infección, con graves consecuencias para la salud del caso índice, situación que explica la alta letalidad 
por tuberculosis. Por otra parte, el prolongado período sin diagnóstico, hace posible un importante número de contagios en contactos domésticos, sociales, laborales y ocasionales. La gran cantidad de infecciones producidas por esta causa, se relacionan con el creciente exceso de morbilidad observada en los años siguientes a la caída en la eficiencia en la pesquisa.

\section{Deficiencias en la red de laboratorios}

Hay debilidad de la red de laboratorios de bacteriología de la tuberculosis por reducción de las horas del profesional destinado a estas funciones y falta de mantenimiento y renovación de infraestructura y equipamiento. En muchos laboratorios se trabaja con material que ya cumplió su vida útil, en otros faltan equipos, y no siempre se dispone de los elementos de bioseguridad.

Si bien en muchos casos hay capacidad ociosa en los laboratorios, por el menor número de muestras que se reciben, si el sistema de localización de casos operase con máxima eficiencia, se harían más evidentes las insuficiencias señaladas. Pese al menor número de muestras, son frecuentes las demoras en entregar los resultados y en ocasiones no se efectúan los exámenes de cultivos indicados.

Todo ello desincentiva a los profesionales de laboratorio, que escasamente tienen tiempo para efectuar los exámenes requeridos, siendo su participación y compromiso con la gestión del PCT con frecuencia nula. Se hace muy difícil asegurar la permanencia de los profesionales en sus funciones y llenar los cargos vacantes.

Pero, tan importante como las deficiencias de la red de laboratorios, ha sido la incapacidad para renovar y perfeccionar las estrategias operacionales para mejorar la cobertura y eficiencia del sistema de localización de casos.

\section{Ineficiencia del tratamiento}

Persiste alta mortalidad e incremento de la tasa de abandono real (abandonos, más traslados sin conocimiento de su egreso final), lo cual afecta severamente la eficiencia del tratamiento. No siempre se ha contado con nuevas estrategias operacionales y disponibilidad de recursos para prevenir la irregularidad del tratamiento y el abandono. No es infrecuente constatar la falta de calidad de la atención para reducir la letalidad en casos graves.

A pesar que la eficacia de los esquemas en uso en estos países es buena, ha habido demora o incapacidad para incorporar tecnologías apropiadas para asegurar el máximo porcentaje de curaciones y plena adherencia al tratamiento.

\section{Recomendaciones}

Las recomendaciones siguientes obligan a los encargados nacionales, de nivel intermedio y locales del PCT, a asumir una actitud proactiva, manteniendo una alta eficiencia en las actividades habituales y la incorporación de aquellas que pueden modificar positivamente la situación actual.

\section{Recuperar la trascendencia de la enfermedad}

Desarrollar, como Programa, un plan de acción proactivo, que permita mejorar la información y conocimiento sobre la epidemiología de la enfermedad, su trascendencia social e importancia de las medidas del Programa de Control y Eliminación de la Tuberculosis. En base a esta actividad, se debe ejercer la abogacía indispensable para incluir la "Eliminación de Tuberculosis como Problema de Salud Pública", como un componente de una política de estado en salud.

Entregar continuamente materiales de información y conocimiento a medios formales de comunicación, como canales de televisión, radios y periódicos y a medios informales, como son las Redes de Comunicación Social, de creciente importancia en la actualidad. Obtener la designación de un integrante del Equipo de Relaciones Públicas del Ministerio que sirva en forma permanente como contraparte informativa del nivel ministerial del PCT, para asegurar un flujo útil de información a la comunidad.

Intentar lograr una participación multisectorial en introducir y ampliar la información y establecer compromisos y objetivos en relación a tuberculosis y su control (Ministerios de Justicia, Educación, Desarrollo social y otros).

La entrega de información y la definición de una política de estado en salud, que considere el objetivo de la eliminación de la tuberculosis, crearían las condiciones y marco de referencia para mantener la voluntad política y toma de decisiones requerida para alcanzar máxima cobertura y eficiencia en las actividades de control, y para cumplir las metas operacionales, epidemiológicas y sanitarias, para lograr la eliminación de la enfermedad.

\section{Mejorar la información del personal de salud sobre la tuberculosis y las bases y procedimientos para su control}

Se debe mejorar el conocimiento que tiene el personal de salud, tanto de profesionales, como auxiliares para-médicos y empleados de servicio, sobre la situación actual de la tuberculosis, la importancia que tienen los casos de tuberculosis 
pulmonar contagiosa que aparecen y existen en la comunidad, las medidas de control disponibles y su racionalidad.

En el ámbito de servicios de salud donde funcionen escuelas de profesiones de la salud, ofrecer clases y talleres sobre este tema, a cargo de los integrantes del equipo local del PCT.

El personal debe ser informado de los resultados de las evaluaciones de Servicios Regionales y Nacionales, relativas al control de la tuberculosis y la calificación de su unidad en ese contexto.

\section{Recuperar el nivel operativo de la red de laboratorios para el control de la Tuberculosis}

A pesar del actual marco de restricción de recursos para el Programa, es prioritario reforzar la red de laboratorios, mejorar sus instalaciones, renovar el equipamiento obsoleto e incorporar nuevas tecnologías apropiadas para mejorar la capacidad de localización de casos y asegurar la calidad diagnóstica. Todo ello contribuirá a atraer, motivar y retener al personal de laboratorio que cumple funciones para el control de la tuberculosis.

\section{Mejorar la localización de casos}

Se debe mantener la actual estrategia de localización de casos de tuberculosis pulmonar en establecimientos de atención ambulatoria y salas de hospitalizaciones, mediante el tamizaje consistente en identificar personas con síntomas respiratorios, tos y expectoración durante más de 2 semanas, entre consultantes y hospitalizados y efectuar estudio bacteriológico (baciloscopía y cultivo), en las muestras de expectoración obtenidas. Deben mantenerse las metas operacionales de cumplimiento de las acciones mediante el uso del indicador de pesquisa (número de baciloscopías diagnósticas/ consultas de morbilidad adulto x $1.000 ;$ meta $=50$ ).

Se debe mejorar la eficiencia en la identificación de consultantes con síntomas respiratorios, simplificar el formulario de solicitud de examen, crear condiciones adecuadas para la obtención de la primera muestra (inmediata) y asegurar la obtención de la segunda muestra en forma diferida.

Estimular el mejor cumplimiento de actividades mediante distinciones al personal y premios a los establecimientos más destacados.

\section{Focalización e intensificación de la localización de casos en grupos vulnerables}

Se deben identificar los grupos de población vulnerables, definidos por el PCT en la población de cada Servicio de Salud y desarrollar en relación a ellos un diagnóstico de situación que per- mita estimar su tamaño y localización geográfica, su estructuración y organización institucional o social.

Con ese conocimiento, y en conjunto con directivos responsables y líderes naturales de estos grupos, planificar acciones para extender, intensificar y profundizar, en forma continua, las medidas de localización, diagnóstico y tratamiento de casos de tuberculosis.

\section{Mejorar eficiencia del tratamiento}

Se deben desarrollar procedimientos para asegurar la adhesión al tratamiento: Ampliar la información inicial que se entrega al enfermo sobre la enfermedad, reforzando la importancia de la adherencia al tratamiento y precisar el perfil psicológico, económico y social del enfermo. Usar esa información como un instrumento de prevención del abandono.

Dar a conocer el riesgo que implica el abandono del tratamiento, tanto para el propio enfermo como para su familia y la comunidad. Desarrollar el concepto que tratar con éxito un caso de tuberculosis es un desafío y una obligación sanitaria, y que equipos de salud de buena calidad tienen mejores resultados en la curación de los enfermos. Distinguir y premiar a estos equipos puede ser de valor como estímulo para el conjunto de la Red Asistencial.

\section{Obtención de recursos financieros}

La inversión en el control de la tuberculosis tiene alta rentabilidad como un medio efectivo de intervención. Se debe conocer el presupuesto anual de tuberculosis, cómo se gasta y qué y cuánto se necesita para mejorar su eficiencia.

\section{Comentario final}

A fines de la década de los ' 60 del siglo pasado, se disponía de esquemas de alta eficacia, se había reconocido el papel fundamental de la bacteriología para el diagnóstico y evaluación del tratamiento y las bases conceptuales y operacionales de éste estaban establecidas. En su tiempo fueron avances sensacionales y es inexcusable no seguir obteniendo actualmente los mejores resultados, optimizando su utilización.

Las señales de alarma sobre creciente ineficiencia del PCT en los países de baja prevalencia de nuestra región, ponen en evidencia la pérdida de estos enfoques. Recuperarlos es de la más alta prioridad. Sin ellos, la introducción de las más modernas tecnologías, por sí solas, no logrará mejorar la operación del Programa ni 
cumplir las metas epidemiológicas y sanitarias propuestas.

La formulación de las estrategias básicas del PCT propiciada por la OMS, hace 40 ó más años, conservan toda su pertinencia. Sin embargo, se hace necesario adecuar aspectos específicos a la situación epidemiológica, demográfica y operacional actual en los países de baja prevalencia, introduciendo nuevas tecnologías apropiadas, y tácticas operacionales innovadoras que mejoren la eficiencia del Programa de Control de la Tuberculosis.

\section{Correspondencia a:}

Dra. Tania Herrera M.

Directora Programa Nacional de Control de la

Tuberculosis, Ministerio de Salud, Chile.

E-mail: tania.herrera@minsal.cl 